\title{
On a Conjecture of Kac-Wakimoto
}

\author{
By
}

Feng $\mathrm{XU}^{*}$

\begin{abstract}
We prove a conjecture about mininmal index of certain representations of Coset Algebraic Conformal Field Theories under certain conditions as formulated previously by us. As a by-product, the Kac-Wakimoto Conjecture (KWC) which is related to the asympotics of the coset characters is true under the same conditions. The same idea in the proof also proves a recent conjecture related to subfactors from conformal inclusions.
\end{abstract}

\section{$\S 1$. Introduction}

Let us first recall some definitions from [X4].

Let $G$ be a simply connected compact Lie group and let $H \subset G$ be a connected subgroup. Denote by $L G$ (cf. [PS]) the group of smooth maps from a circle to $G$. Let $\pi^{i}$ be an irreducible representations of $L G$ with positive energy at level $k^{1}$ on Hilbert space $H^{i}$. Suppose when restricting to $L H, H^{i}$ decomposes as:

$$
H^{i}=\sum_{\alpha} H_{i, \alpha} \otimes H_{\alpha}
$$

and $\pi_{\alpha}$ are irreducible representations of $L H$ on Hilbert space $H_{\alpha}$. The set of $(i, \alpha)$ which appears in the above decompositions will be denoted by exp.

To illustrate the new ideas in this paper we will focus on the case when both $G$ and $H$ are simply connected semisimple compact Lie groups of type $A$, i.e., $G$ and $H$ are of the forms $S U\left(N_{1}\right) \times S U\left(N_{2}\right) \times \ldots \times S U\left(N_{n}\right)$. The ideas of

Communicated by M. Kashiwara, March 14, 2000.

1991 Mathematics Subject Classification(s): 46S99, 81R10.

This work is partially supported by NSF grant DMS-9820935.

* Department of Mathematics, 2208 Sproul Hall, University of California at Riverside, Riverside, CA 92521, U.S.A.

e-mail: xufeng@math.ucr.edu 
this paper can be applied to all compact semisimple and simply connected Lie groups and we plan to consider them in separate publications.

We shall use $\pi^{1}$ (resp. $\left.\pi_{1}\right)^{2}$ to denote the vacuum representation of $L G$ (resp. $L H)$. Let $\mathcal{A}_{G / H}$ be the irreducible conformal precosheaf of the coset $G / H$ as defined in Prop. 2.2 of [X4]. The decompositions above naturally give rise to a class of covariant representations of $\mathcal{A}$, denoted by $\pi_{i, \alpha}$ or simply $(i, \alpha)$. By Th. 2.3 of $[\mathrm{X} 4], \pi_{1,1}$ is the vacuum representation of $\mathcal{A}_{G / H}$.

Let us denote by $S_{i j}$ (resp. $S_{\alpha \beta}$ ) the $S$ matrices of $L G$ (resp. $L H$ ) at level $k$ (resp. certain level of $L H$ determined by the inclusion $H \subset G_{k}$ ) as defined on P. 264 of [Kac]. Define ${ }^{3}$

$$
b(i, \alpha)=\sum_{(j, \beta)} S_{i j} \overline{\dot{S}_{\alpha \beta}}\langle(j, \beta),(1,1)\rangle
$$

Note the above summation is effectively over those $(j, \beta)$ such that $(j, \beta) \in \exp$. The definition of $\langle x, y\rangle$ for any two sectors $x, y$ can be found in Section 2.1 before formula (0).

The Kac-Wakimoto Conjecture (KWC) states that if $(i, \alpha) \in \exp$, then $b(i, \alpha)>0$ (cf. Conj. 2.5 of $[\mathrm{KW}])$. This conjecture is related to the asymptotics of the coset characters (cf. Th. B of $[\mathrm{KW}]$ ).

In Section 2.4 of [X4] an even stronger conjecture, Conjecture 2 (C2) is formulated. This conjecture states that the square root of the minimal index (cf. Section 2.1) of sector $(i, \alpha)$, denoted by $d_{(i, \alpha)}$, is given by

$$
d_{(i, \alpha)}=\frac{b(i, \alpha)}{b(1,1)}
$$

$\mathrm{C} 2$ is stronger than $\mathrm{KWC}$ since $d_{(i, \alpha)} \geq 1, b(1,1)>0$.

In [X4], C2 is proved for a class of cosets, but the proof is based on the known results about the branching rules, which already implies KWC. The main improvement in this paper is a proof of $\mathrm{C} 2$ under general conditions, without knowing details about the branching rules (cf. Th. 3.4). The power of the new ideas in this paper can be seen from the examples listed in Section 3.1 after Cor. 3.5, where we show that KWC is true for infinite series of cosets which do not seem to have been obtained by other methods. We also give a simple proof of a conjecture (Conj. 7.1) in [BE3]. ${ }^{4}$

Let us describe the content of this paper in more details. In Section 2 we collect some results from [Reh] and [KLM] which will be used in the proof of Prop. 3.1 in Section 3. In particular the notion of modular matrices from [Reh], and the notion of $\mu$-index from [KLM] are introduced. In Lemma 2.2 we calculate the $\mu$-index of the coset. Lemma 2.3 is an application of Prop. 3.1 
of [BEK2] and Prop. 3.1 of [BE4] to our setting. In Prop. 2.4, we show that the global index of the coset is the same as its $\mu$-index using Lemma 2.3. This implies the non-degeneracy of the modular matrices for the coset under the conditions of Prop. 2.4 by Cor. 32 and Th. 38 of [KLM]. We also give a second proof of this result by using relative braidings first used in [X1] and studied in details in [BE3], [BEK1]. The $n$-regularity (cf. definition in Section 2.2) of our coset (Cor. 2.5) follows from Lemma 2.3 and Cor. 7 of [KLM].

The proof of Prop. 3.1 contains one of the new ideas of this paper, which is a novel way of calculating the summation on the right-hand side of the equation in (1) of Prop. 3.1. Prop. 3.1 is then used to prove Cor. 3.2, Cor. 3.3, Th. 3.4 and Cor. 3.5. The main results of Section 3, Th. 3.4 and Cor. 3.5 have already been described at the beginning of this introduction. The same idea in the proof of Prop. 3.1 is also used in Section 3.2 to give a proof of Conj. 7.1 in [BE3] (Th. 3.7).

As we already noted before, all the Lie groups considered in this paper will be simply connected semisimple compact Lie group of type $A$, i.e., groups of the form $S U\left(N_{1}\right) \times S U\left(N_{2}\right) \times \ldots \times S U\left(N_{n}\right)$ unless stated otherwise.

\section{Acknowledgement}

I'd like to thank Professor Y. Kawahigashi for sending me [KLM].

\section{$\S 2 . \quad$ Preliminaries}

\section{§2.1. Genus 0 and 1 modular matrices}

Let us first recall some definitions from [X2]. Let $M$ be a properly infinite factor and $\operatorname{End}(M)$ the semigroup of unit preserving endomorphisms of $M$. In this paper $M$ will always be the unique hyperfinite $I I I_{1}$ factors. Let $\operatorname{Sect}(M)$ denote the quotient of $\operatorname{End}(M)$ modulo unitary equivalence in $M$. We denote by $[\rho]$ the image of $\rho \in \operatorname{End}(M)$ in $\operatorname{Sect}(M)$.

It follows from [L3] and [L4] that $\operatorname{Sect}(M)$, with $M$ a properly infinite von Neumann algebra, is endowed with a natural involution $\theta \rightarrow \bar{\theta}$; moreover, $\operatorname{Sect}(M)$ is a semiring with identity denoted by $i d$ or 1 when no confusion arises.

If given a normal faithful conditional expectation $\epsilon: M \rightarrow \rho(M)$, we define a number $d_{\epsilon}$ (possibly $\infty$ ) by:

$$
d_{\epsilon}^{-2}:=\operatorname{Max}\left\{\lambda \in[0,+\infty) \mid \epsilon\left(m_{+}\right) \geq \lambda m_{+}, \forall m_{+} \in M_{+}\right\}
$$

(cf. $[\mathrm{PP}])$. 
We define

$$
d=\operatorname{Min}_{\epsilon}\left\{d_{\epsilon} \mid d_{\epsilon}<\infty\right\} .
$$

$d$ is called the statistical dimension of $\rho$. It is clear from the definition that the statistical dimension of $\rho$ depends only on the unitary equivalence classes of $\rho$. The properties of the statistical dimension can be found in [L1], [L3] and [L4]. We will denote the statistical dimension of $\rho$ by $d_{\rho}$ in the following. $d_{\rho}^{2}$ is called the minimal index of $\rho$.

Recall from [X2] that we denote by $\operatorname{Sect}_{0}(M)$ those elements of $\operatorname{Sect}(M)$ with finite statistical dimensions. For $\lambda, \mu \in \operatorname{Sect}_{0}(M)$, let $\operatorname{Hom}(\lambda, \mu)$ denote the space of intertwiners from $\lambda$ to $\mu$, i.e. $a \in \operatorname{Hom}(\lambda, \mu)$ iff $a \lambda(x)=\mu(x) a$ for any $x \in M . \operatorname{Hom}(\lambda, \mu)$ is a finite dimensional vector space and we use $\langle\lambda, \mu\rangle$ to denote the dimension of this space. $\langle\lambda, \mu\rangle$ depends only on $[\lambda]$ and $[\mu]$. Moreover we have

$$
\langle\nu \lambda, \mu\rangle=\langle\lambda, \bar{\nu} \mu\rangle,\langle\nu \lambda, \mu\rangle=\langle\nu, \mu \bar{\lambda}\rangle
$$

which follows from Frobenius duality (See [L2]).

Next we will recall some of the results of [Reh] (also cf. [FRS]) and introduce notations.

Let $\left\{\left[\rho_{i}\right], i \in I\right\}$ be a finite set of equivalence classes of irreducible covariant representations of an irreducible conformal precosheaf (cf. Section 2.1 of [GL]). Suppose this set is closed under conjugation and composition. We will denote the conjugate of $\left[\rho_{i}\right]$ by $\left[\rho_{\bar{i}}\right]$ and identity sector by [1] if no confusion arises, and let $N_{i j}^{k}=\left\langle\left[\rho_{i}\right]\left[\rho_{j}\right],\left[\rho_{k}\right]\right\rangle$. We will denote by $\left\{T_{e}\right\}$ a basis of isometries in $\operatorname{Hom}\left(\rho_{k}, \rho_{i} \rho_{j}\right)$. The univalence of $\rho_{i}$ (cf. P.12 of [GL]) will be denoted by $\omega_{\rho_{i}}$.

Let $\phi_{i}$ be the unique minimal left inverse of $\rho_{i}$, define:

$$
Y_{i j}:=d_{\rho_{i}} d_{\rho_{j}} \phi_{j}\left(\epsilon\left(\rho_{j}, \rho_{i}\right)^{*} \epsilon\left(\rho_{i}, \rho_{j}\right)^{*}\right),
$$

where $\epsilon\left(\rho_{j}, \rho_{i}\right)$ is the unitary braiding operator (cf. [GL]).

We list two properties of $Y_{i j}$ (cf. (5.13), (5.14) of [Reh]) which will be used in Section 2.2:

$$
\begin{aligned}
& Y_{i j}=Y_{j i}=Y_{i \bar{j}}^{*}=Y_{\bar{i} \bar{j}} \\
& Y_{i j}=\sum_{k} N_{i j}^{k} \frac{\omega_{i} \omega_{j}}{\omega_{k}} d_{\rho_{k}}
\end{aligned}
$$

Define $\tilde{\sigma}:=\sum_{i} d_{\rho_{i}}^{2} \omega_{\rho_{i}}^{-1}$. If the matrix $\left(Y_{i j}\right)$ is invertible, by Proposition on P.351 of [Reh] $\tilde{\sigma}$ satisfies $|\tilde{\sigma}|^{2}=\sum_{i} d_{\rho_{i}}^{2}$. Suppose $\tilde{\sigma}=|\tilde{\sigma}| \exp (i x), x \in \mathbb{R}$. Define matrices

$$
S:=|\tilde{\sigma}|^{-1} Y, T:=C \operatorname{Diag}\left(\omega_{\rho_{i}}\right)
$$


where $C:=\exp \left(i \frac{x}{3}\right)$. Then these matrices satisfy the algebra:

$$
\begin{aligned}
& S S^{\dagger}=T T^{\dagger}=i d, \\
& T S T S T=S, \\
& S^{2}=\hat{C}, T \hat{C}=\hat{C} T=T,
\end{aligned}
$$

where $\hat{C}_{i j}=\delta_{i \bar{j}}$ is the conjugation matrix. Moreover

$$
N_{i j}^{k}=\sum_{m} \frac{S_{i m} S_{j m} S_{k m}^{*}}{S_{1 m}} .
$$

(7) is known as Verlinde formula.

We will refer the $S, T$ matrices as defined in (3) as genus 0 modular matrices since they are constructed from the fusions rules, monodromies and minimal indices which can be thought as genus 0 data associated to a Conformal Field Theory (cf. [MS]).

It follows from (7) and (4) that any irreducible representation of the commutative ring generated by $i$ 's is of the form $i \rightarrow \frac{S_{i j}}{S_{1 j}}$.

Now let us consider an example which verifies (1) to (7) above. Let $G=$ $S U(N)$. We denote $L G$ the group of smooth maps $f: S^{1} \mapsto G$ under pointwise multiplication. The diffeomorphism group of the circle Diff $S^{1}$ is naturally a subgroup of $\operatorname{Aut}(L G)$ with the action given by reparametrization. In particular the group of rotations $\operatorname{Rot} S^{1} \simeq U(1)$ acts on $L G$. We will be interested in the projective unitary representation $\pi: L G \rightarrow U(H)$ that are both irreducible and have positive energy. This means that $\pi$ should extend to $L G \ltimes \operatorname{Rot} S^{1}$ so that $H=\oplus_{n \geq 0} H(n)$, where the $H(n)$ are the eigenspace for the action of $\operatorname{Rot} S^{1}$, i.e., $r_{\theta} \xi=\exp ^{i n \theta}$ for $\theta \in H(n)$ and $\operatorname{dim} H(n)<\infty$ with $H(0) \neq 0$. It follows from [PS] that for fixed level $K$ which is a positive integer, there are only finite number of such irreducible representations indexed by the finite set

$$
P_{++}^{h}=\left\{\lambda \in P \mid \lambda=\sum_{i=1, \cdots, N-1} \lambda_{i} \Lambda_{i}, \lambda_{i} \geq 1, \sum_{i=1, \cdots, n-1} \lambda_{i}<h\right\}
$$

where $P$ is the weight lattice of $S U(N)$ and $\Lambda_{i}$ are the fundamental weights and $h=N+K$. We will use 1 to denote the trivial representation of $S U(N)$. For $\lambda, \mu, \nu \in P_{++}^{K}$, define

$$
N_{\lambda \mu}^{\nu}=\sum_{\delta \in P_{++}^{K}} \frac{S_{\lambda \delta} S_{\mu \delta} S_{\nu \delta}^{*}}{S_{1 \delta}}
$$


where $S_{\lambda \delta}$ is given by the Kac-Peterson formula:

$$
S_{\lambda \delta}=c \sum_{w \in S_{N}} \varepsilon_{w} \exp (i w(\delta) \cdot \lambda 2 \pi / n) .
$$

Here $\varepsilon_{w}=\operatorname{det}(w)$ and $c$ is a normalization constant fixed by the requirement that $\left(S_{\lambda \delta}\right)$ is an orthonormal system. It is shown in [Kac] P. 288 that $N_{\lambda \mu}^{\nu}$ are non-negative integers. Moreover, define $\operatorname{Gr}\left(C_{K}\right)$ to be the ring whose basis are elements of $P_{++}^{K}$ with structure constants $N_{\lambda \mu}^{\nu}$. The natural involution $*$ on $P_{++}^{K}$ is defined by $\lambda \mapsto \lambda^{*}=$ the conjugate of $\lambda$ as representation of $S U(N)$. All the irreducible representations of $\operatorname{Gr}\left(C_{K}\right)$ are given by $\lambda \rightarrow \frac{S_{\lambda \mu}}{S_{1 \mu}}$ for some $\mu$.

The irreducible positive energy representations of $L S U(N)$ at level $K$ give rise to an irreducible conformal precosheaf $\mathcal{A}_{G}$ and its covariant representations by the results in Section 17 of [W2]. $\mathcal{A}_{G}$ is a collection of maps $I \in \mathcal{I} \rightarrow \mathcal{A}_{G}(I)$ from the proper intervals on a circle to von Neumann algebras which satifsy conditions as defined in Section 2 of [GL], and one can also find the definitions of covariant representations of $\mathcal{A}_{G}$ is Section 2 of [GL]. We will use $\lambda$ to denote such representations.

For $\lambda$ irreducible, the univalence $\omega_{\lambda}$ is given by an explicit formula. Let us first define

$$
\Delta_{\lambda}=\frac{c_{2}(\lambda)}{K+N}
$$

where $c_{2}(\lambda)$ is the value of Casimir operator on representation of $S U(N)$ labeled by dominant weight $\lambda$ (cf. 1.4 .1 of $[\mathrm{KW}]$ ). $\Delta_{\lambda}$ is usually called the conformal dimension. Then we have $\omega_{\lambda}=\exp \left(2 \pi i \Delta_{\lambda}\right)$. Note that $\omega_{\lambda}=\omega_{\bar{\lambda}}$.

Define the central charge (cf. 1.4.2 of $[\mathrm{KW}]$ )

$$
C_{G}:=\frac{K \operatorname{dim}(\mathrm{G})}{K+N}
$$

and $T$ matrix as

$$
T=\operatorname{diag}\left(\dot{\omega}_{\lambda}\right)
$$

where $\dot{\omega}_{\lambda}=\omega_{\lambda} \exp \left(\frac{-2 \pi i C_{G}}{24}\right)$. By Th. 13.8 of [Kac] $S$ matrix as defined in (9) and $T$ matrix in (12) satisfy relation (4), (5) and (6). Since $S, T$ matrix defined in (8) and (11) are related to the modular properties of characters which are related to Genus 1 data of CFT (cf. [MS]), we shall call them genus 1 modular matrices. 
By Cor. 1 in Section 34 of [W2], The fusion ring generated by all $\lambda \in P_{++}^{(K)}$ is isomorphic to $\operatorname{Gr}\left(C_{K}\right)$, with structure constants $N_{\lambda \mu}^{\nu}$ as defined in (8). One may therefore ask what are the $Y$ matrix (cf. (0)) in this case. By using (2) and the formula for $N_{\lambda \mu}^{\nu}$, a simple calculation shows:

$$
Y_{\lambda \mu}=\frac{S_{\lambda \mu}}{S_{1 \mu}},
$$

and it follows that $Y_{\lambda \mu}$ is nondegenerate, and $S, T$ matrices as defined in (3) are indeed the same $S, T$ matrix defined in (8) and (11), which is a surprising fact. This fact is refered to as genus 0 modular matrices coincide with genus 1 modular matrices. If the analogue of Cor. 1 in Section 34 of [W2] is established for other types of simple and simply connected Lie groups, then this fact is also true for other types of groups by the same argument.

\section{$\S 2.2$. Nondegeneracy of the coset}

Let $H \subset G_{k}$ be as in the introduction. We will use $\mathcal{A}_{G}, \mathcal{A}_{H}$ to denote the irreducible conformal precosheaves associated with $G$ and $H$ respectively (see paragraph before (10) in Section 2.1). Denote by $\mathcal{A}_{G / H}$ the irreducible conformal precosheaves associated with the coset $H \subset G_{k}$ as defined in Prop. 2.2 of [X4].

In [X4], certain rationality results (cf. Th. 4.2) are proved for a class of coset $H \subset G_{k}$. A stronger rationality condition, $\mu$-rational or completely rational, is defined in Section 3 of [KLM], and we will recall these definitions.

Let $\mathcal{A}$ be an irreducible conformal precosheaf on a circle $S^{1}$. Two proper intervals $I_{1}, I_{2}{ }^{5}$ of the circle are said to be disjoint if $\bar{I}_{1} \cap \bar{I}_{2}=\emptyset$. Denote by $\mathcal{E}_{2}$ the set of two disjoint intervals. $\mathcal{A}$ is said to be split if $\mathcal{A}\left(I_{1}\right) \vee \mathcal{A}\left(I_{2}\right)$ is naturally isomorphic to the tensor product of von Neumann algebras $\mathcal{A}\left(I_{1}\right) \otimes \mathcal{A}\left(I_{2}\right)$ for all $\left\{I_{1}, I_{2}\right\} \in \mathcal{E}_{2}$.

Let $\left\{I_{1}, I_{2}\right\} \in \mathcal{E}_{2}$, and let $I_{3}, I_{4}$ be the disjoint intervals such that $I_{3} \cup I_{4}$ is the interior of the complement of $I_{1} \cup I_{2}$ in $S^{1}$. If the index

$$
\left[A\left(I_{3}^{\prime}\right) \cap A\left(I_{4}^{\prime}\right): \mathcal{A}\left(I_{1}\right) \vee \mathcal{A}\left(I_{2}\right)\right]
$$

is independent of $\left\{I_{1}, I_{2}\right\} \in \mathcal{E}_{2}$, then this index is called the $\mu$-index of $\mathcal{A}$, denoted by $\mu_{\mathcal{A}}$. $\mathcal{A}$ is said to be completely rational, or $\mu$-rational for short, if $\mathcal{A}$ is strongly additive with finite $\mu$-index.

Recall that $\mathcal{A}$ is strongly additive if $\mathcal{A}\left(I_{1}\right) \vee \mathcal{A}\left(I_{2}\right)=\mathcal{A}(I)$ where $I_{1} \cup I_{2}$ is obtained by removing an interior point from $I$. $\mathcal{A}$ is said to be $n$-regular if 
$\mathcal{A}\left(S^{1}-\left\{p_{1}, \ldots, p_{n}\right\}\right)=\mathcal{A}\left(S^{1}\right)$ for any $p_{1}, \ldots, p_{n} \in S^{1}$. Note that if $\mathcal{A}$ is strongly additive, then $\mathcal{A}$ is $n$-regular for any $n$.

Here we will make corrections on a statement concerning strong additivity in [X4]. When $G$ is of type $A$, it is proved (cf. Remark on P. 504 of [W2] and [W4]) that $\mathcal{A}_{G}$ is strongly additive. But as pointed out to us by Prof. A. Wassermann, the proof of this fact, Th. E in P. 504 of [W2], is not correct (This does not affect the results of [W2] ). It follows that the proof of (1) of Lemma 2.1 and the proof of a remark on strong additivity at the end of Section 2.1 in [X4] which follows from the proof of Th. E in [W2] is not correct. We note that the correct proof of Th. E as remarked in [W2] (also cf. [TL]) applies without change to give a proof of (1) of Lemma 2.1 in [X4]. The remark on strong additivity at the end of Section 2.1 in [X4] is used in Section 4 of [X4] only to ensure the equivalence of local and global intertwinners (also cf. Section 2.3 of [BE1]). More precisely, the equivalence of local and global intertwinners is used in the proof of Braiding-Fusion equations. But under the condition of finite index and conformal invariance, the equivalence of local and global intertwinners has been proved in Th. 2.3 of [GL]. Hence all the lemmas, corollaries and theorems of [X2] hold without the strong additivity assumption since one can instead use Th. 2.3 of [GL]. However it will be interesting to prove strong additivity for the net associated with the coset. See Cor. 2.5 for a positive result in this direction. We will show in Prop. 2.4 how one can still get nondegeneracy of modular matrices for the coset without knowing strong additivity.

When $\mathcal{A}$ has finite $\mu$-index, then $\mathcal{A}$ has only finitely many irreducible covariant representations with finite index (cf. Th. 8 of $[\mathrm{KLM}]$ and the remark after it), denoted by $\rho_{i}, i=1, \ldots, n$. The global index of $\mathcal{A}$, denoted by $I_{\mathcal{A}}$, is defined to be $I_{\mathcal{A}}:=\sum_{i} d_{\rho_{i}}^{2}$. Note that by the proof of Th. 38 in [KLM] (also cf. Section 3 of $[\mathrm{X} 3]) I_{\mathcal{A}} \leq \mu_{\mathcal{A}}$.

Denote by $\mu_{G}, \mu_{H}, \mu_{G / H}$ the $\mu$ indices of the irreducible conformal precosheaves $\mathcal{A}_{G}, \mathcal{A}_{H}$ and $\mathcal{A}_{G / H}$ associated with $H, G$ and the coset $H \subset G$ respectively. The irreducible covariant representations of $\mathcal{A}_{G}, \mathcal{A}_{H}$ and $\mathcal{A}_{G / H}$ will be denoted by $i, \alpha$ and $x$ respectively. For simplicity the global index of $\mathcal{A}_{G}, \mathcal{A}_{H}$ and $\mathcal{A}_{G / H}$ will be denoted by $I_{G}, I_{H}$ and $I_{G / H}$ respectively. Note that as in Section $1(1,1)$ will denote the vacuum representation of $\mathcal{A}_{G / H}$. As in Section 1 , let $\pi^{1}$ denote the vacuum representation of $\mathcal{A}_{G}$. Then one has a natural inclusion (cf. Section 3 of [X4])

$$
\pi^{1}\left(\mathcal{A}_{G / H}(I) \otimes \mathcal{A}_{H}(I)\right) \subset \mathcal{A}_{G}(I)
$$

for any proper interval $I$ of a circle. The coset $H \subset G_{k}$ is cofinite if the above 
inclusion has finite index (cf. Section 3 of [X4]), and the square root of this index is denoted by $d(G / H)$. As noted in Section 3 of $[\mathrm{X} 4], d(G / H)$ is independent of the choices of $I$. By (3.1) of [X4], we have

$$
d(G / H)^{2}=\sum_{\alpha} d_{(1, \alpha)} d_{\alpha}
$$

where $d_{(1, \alpha)}$ and $d_{\alpha}$ are the statistical dimensions of covariant representations $(1, \alpha)$ and $\alpha$ respectively.

We have:

Lemma 2.2. $\quad$ Suppose that $H \subset G_{k}$ is cofinite, and $\mathcal{A}_{H}$ and $\mathcal{A}_{G}$ are $\mu$-rational. Then $\mathcal{A}_{G / H}$ is split and has finite $\mu$ index. In fact

$$
\mu_{G / H}=\frac{d(G / H)^{4} \mu_{G}}{\mu_{H}}
$$

Proof. First note that $\mathcal{A}_{G / H}$ is split. This is a well known fact which follows from the asymptotics of the growth of states in the vaccum (cf. Th. B of $[\mathrm{KW}])$ and [BAF]. For a simplified proof see Prop. 2.3.1 of [X7] which follows from [W3]. Then the $\mu$-index of the tensor product of $\mathcal{A}_{H}$ and $\mathcal{A}_{G / H}$ is $\mu_{H} \mu_{G / H}$, and it follows from the proof of Prop. 21 of [KLM] (also cf. the proof of Th. 3.5 in [X3]) that:

$$
\mu_{H} \mu_{G / H}=d(G / H)^{4} \mu_{G}
$$

It follows that

$$
\mu_{G / H}=\frac{d(G / H)^{4} \mu_{G}}{\mu_{H}}
$$

is finite.

Let us note the following interesting consequence of Lemma 2.2. For the diagonal inclusions of type A considered in Section 2.2 of [X5], a direct calculation using Lemma 2.2 shows that

$$
\mu_{G / H}=|\tilde{\sigma}|^{2},
$$

where $\tilde{\sigma}$ is determined in (2) of Th. 2.3 in [X5]. By Th. 38 of [KLM], this shows that the irreducible sectors which are determined in Section 2.2 of [X5] are all the irreducible sectors of the coset theory, and by Cor. 7 of $[\mathrm{KLM}] \mathcal{A}_{G / H}$ is $n$-regular for any $n$. We will see more general statements in Cor. 2.5 and Cor. 3.2. 
The $\mu$-rationality of the irreducible conformal precosheaf associated with a type A group $G$ follows from the results of [W2] and [X3]. In this case one has $I_{G}=\mu_{G}$. The proof of finite $\mu$ index and the calculation of the index value in [X3] are based on the existence of a class of conformal inclusions which exist for all classical simply connected Lie groups.

For the rest of this section, we assume $d(G / H)<\infty$. By Lemma 2.2 and the remark after Th. 8 of $[\mathrm{KLM}], \mathcal{A}_{G / H}$ has only finite number of irreducible covariant representations. In fact the global index $I_{G / H} \leq \mu_{G / H}$. Also note that the set of irreducible covariant representations is closed under conjugation and composition (cf. [GL]). Note that if $x, \alpha$ are the irreducible covariant representations of $\mathcal{A}_{G / H}$ and $\mathcal{A}_{H}$ respectively, then $x \otimes \alpha$ is an irreducible covariant representation of $\mathcal{A}_{G / H} \otimes \mathcal{A}_{H}$. We can take the finite set consisting of $x \otimes \alpha$ where $x, \alpha$ are the irreducible covariant representations of $\mathcal{A}_{G / H}$ and $\mathcal{A}_{H}$ respectively and define the $Y$-matrix as in (0) of Section 2.1. Then one has

$$
Y_{x \otimes \alpha, y \otimes \beta}=Y_{x y} Y_{\alpha \beta},
$$

where $Y_{x y}, Y_{\alpha \beta}$ are the $Y$-matrix associated with the set of irreducible covariant representations of $\mathcal{A}_{G / H}$ and $\mathcal{A}_{H}$ respectively. $Y_{x y}$ will be referred to as the $Y$-matrix of the coset.

In Section 4.2 of [X4], the results of [X1] (also cf. [BE1-2]) are applied to the net of inclusions $\pi^{1}\left(\mathcal{A}_{G / H}(I) \otimes \mathcal{A}_{H}(I)\right) \subset \mathcal{A}_{G}(I)$. The key observation is that there is a ring homomorphism $x \otimes \alpha \rightarrow a_{x \otimes \alpha}$ with certain remarkable properties first established in [X1]. We will refer to Section 4.2 of [X4] for the definition of $a_{x \otimes \alpha}$ and $\sigma_{i}$. A useful property which follows from Prop. 4.2 and (4) of Th. 4.1 of $[\mathrm{X} 4]$ is

$$
\left\langle\sigma_{i}, a_{x \otimes \alpha}\right\rangle=\langle(i, \alpha), x\rangle
$$

So the map $x \rightarrow a_{x \otimes 1}$ as defined in [X4] is a ring isomorphism by (1) of Prop. 4.2 in $[\mathrm{X} 4]$, and we have

$$
\langle x, y\rangle=\left\langle a_{x \otimes 1}, a_{y \otimes 1}\right\rangle .
$$

We will use the notations of Section 4.2 of [X4] and ideas of [X1]. We denote the set of irreducible sectors of $a_{x \otimes \lambda}$ by $W .{ }^{6} \quad$ Notice $\sigma_{i} \in W$, and are referred to as "special nodes" in Section 3.4 of [X1]. The ring homomorphism $x \otimes \alpha \rightarrow a_{x \otimes \alpha}$, up to a unitary equivalence, is called $\alpha$-induction in $[\mathrm{BE} 1,2,3]$. A dictionary between the notations of [X1] and [BE1,2,3] can be found in Section 2.1 of [X6]. If one choose the opposite braiding compared to the braiding in the definition of $a_{x \otimes \alpha}$, one obtain $\tilde{a}_{x \otimes \alpha}$. Let $\tilde{W}$ be the set of 
irreducible subsectors of $\tilde{a}_{x \otimes \alpha}, \forall x, \alpha$. Let $\hat{W}$ be the set of irreducible subsectors of $\tilde{a}_{x \otimes \alpha} a_{y \otimes \beta}, \forall x, \alpha, y, \beta$. Let $W_{0}:=W \cap \tilde{W}$. For any finite set $Z$ of irreducible sectors closed under multiplication we denote by $I_{Z}:=\sum_{\theta \in Z} d_{\theta}^{2}$, where $d_{\theta}$ is the statistical dimension of $\theta$ (cf. Section 2.1). If no possible confusion arises, we will denote the vector space over $\mathbb{C}$ with basis $Z$ by $Z$. This vector space is endowed with an inner product by extending the bilinear form $\langle$,$\rangle on sectors$ (cf. Section 2.1) linearily in the first variable and conjugate linearily in the second variable. Note that every sector of $Z$ gives rise to a linear operator acting on the vector space $Z$ where the action is given by left multiplication.

The following Lemma follows from Prop. 3.1 of [BE4] and Prop. 3.1 of [BEK2]. We include the proof for our case.

\section{Lemma 2.3.}

$$
I_{W}=I_{\tilde{W}}=\frac{I_{H} I_{G / H}}{d(G / H)^{2}}, \quad I_{\hat{W}}=I_{H} I_{G / H}, \quad I_{W_{0}}=\frac{I_{H} I_{G / H}}{d(G / H)^{4}} .
$$

Proof. Each sector $a_{x \otimes \alpha}$ (and linear combinations of them) can be thought as an operator on $W$ where the action is given by left multiplication. These operators $a_{x \otimes \alpha}$ share a common eigenvector $d=\sum_{\lambda \in W} d_{\lambda} \lambda$ where $\lambda$ are elements in the basis $W$, with eigenvalues $d_{x} d_{\alpha}$. Note that the matrix corresponding to $N=\sum_{x \otimes \alpha} a_{x \otimes \alpha}$ on the basis $W$ is irreducible since each element of $W$ is a subsector of some $a_{x \otimes \alpha}$, and $d=\sum_{\lambda \in W} d_{\lambda} \lambda$ is also a Perron-Frobenius eigenvector of $N$. Now define another vector

$$
v:=\sum_{x, \alpha} d_{x} d_{\alpha} a_{x \otimes \alpha}=\sum_{x, \alpha, \lambda} d_{x} d_{\alpha}\left\langle a_{x \otimes \alpha}, \lambda\right\rangle \lambda
$$

Note that $v$ has positive entries under the basis $W$. Since

$$
\begin{aligned}
a_{y \otimes \beta} v=\sum_{x, \alpha} d_{x} d_{\alpha} a_{y \otimes \beta} a_{x \otimes \alpha} & =\sum_{x, z, \alpha, \delta} d_{x} d_{\alpha} N_{x y}^{z} N_{\alpha \beta}^{\delta} a_{z \otimes \delta} \\
& =\sum_{z, \delta} d_{y} d_{\beta} d_{\delta} d_{z} a_{z \otimes \delta} \\
& =d_{y} d_{\beta} \sum_{z, \delta} d_{z} d_{\delta} a_{z \otimes \delta}
\end{aligned}
$$

where we have used the homomorphism property of the map $x \otimes \alpha \rightarrow a_{x \otimes \alpha}$. It follows that $v$ is also an Perron-Frobenius eigenvector of $N$. So there exists a positive constant $c$ such that $v=c d$. By computing the statistical dimension we get $I_{H} I_{G / H}=c I_{W}$. From $\langle v, i d\rangle=c\langle d, i d\rangle$ where $i d$ is the identity sector 
we get (using $d_{i d}=1$ )

$$
c=\sum_{x, \alpha} d_{x} d_{\alpha}\left\langle a_{x \otimes \alpha}, i d\right\rangle=\sum_{x, \alpha} d_{x} d_{\alpha}\langle x,(1, \alpha)\rangle=\sum_{\alpha} d_{(1, \alpha)} d_{\alpha}=d(G / H)^{2}
$$

where in the last equality we have used (13).

Similarly if we define vectors $v^{-}=\sum_{x, \alpha} d_{x} d_{\alpha} \tilde{a}_{x \otimes \alpha}, d^{-}=\sum_{\lambda \in \tilde{W}} d_{\lambda}$, then a similar proof as above shows that $v^{-}=c d^{-}$and $I_{H} I_{G / H}=c I_{\tilde{W}}$. This proves the first equation in the lemma.

To prove the second equation, consider $a_{x \otimes \alpha} \tilde{a}_{y \otimes \beta}$ as operators on $\hat{W}$ with the action given by multiplication on the left. Let $\hat{d}=\sum_{\lambda \in \tilde{W}} d_{\lambda}$. $\hat{d}$ is a common eigenvector of $a_{x \otimes \alpha} \tilde{a}_{y \otimes \beta}$ with eigenvalues $d_{x} d_{\alpha} d_{y} d_{\beta}$. Define another vector

$$
\hat{v}:=\sum_{x, y, \alpha, \beta} d_{x} d_{\alpha} d_{y} d_{\beta} a_{x \otimes \alpha} \tilde{a}_{y \otimes \beta} .
$$

One checks easily using the fact that as sectors $a_{x \otimes \alpha} \tilde{a}_{y \otimes \beta}=\tilde{a}_{y \otimes \beta} a_{x \otimes \alpha}$ (cf. Lemma 3.3 of [X1] or [BE2]) that $\hat{v}$ is also a common eigenvector of $a_{x \otimes \alpha} \tilde{a}_{y \otimes \beta}$ with eigenvalues $d_{x} d_{\alpha} d_{y} d_{\beta}$. Let $\hat{N}:=\sum_{x, y, \alpha, \beta} a_{x \otimes \alpha} \tilde{a}_{y \otimes \beta}$. Then the matrix of $\hat{N}$ under the basis $\hat{W}$ is irreducible since every irreducible sector in $\hat{W}$ appears as a descendant of some $a_{x \otimes \alpha} \tilde{a}_{y \otimes \beta}$. It follows that $\hat{d}, \hat{v}$ are Perron-Frobenius eigenvectors of $\hat{N}$, and so $\hat{v}=\hat{c} \hat{d}$ for some positive constant $\hat{c}$. By computing the statistical dimension we get: $\left(I_{H} I_{G / H}\right)^{2}=\hat{c} I_{\hat{W}}$. From $\langle\hat{v}, i d\rangle=\hat{c}\langle\hat{d}, i d\rangle$ we get (using $d_{i d}=1$ )

$$
\begin{aligned}
\hat{c}=\langle\hat{v}, i d\rangle & =\sum_{x, y, \alpha, \beta} d_{x} d_{\alpha} d_{y} d_{\beta}\left\langle a_{x \otimes \alpha} \tilde{a}_{y \otimes \beta}, i d\right\rangle \\
& =\sum_{x, y, \alpha, \beta} d_{x} d_{\alpha} d_{y} d_{\beta}\left\langle a_{x \otimes \alpha}, \tilde{a}_{\bar{y} \otimes \bar{\beta}}\right\rangle \\
& =\sum_{x, y, \alpha, \beta} d_{x} d_{\alpha} d_{y} d_{\beta}\left\langle a_{x \otimes \alpha}, \tilde{a}_{y \otimes \beta}\right\rangle \\
& =\sum_{x, y, \alpha, \beta} Y_{x \otimes \alpha,(1,1) \otimes 1}\left\langle a_{x \otimes \alpha}, \tilde{a}_{y \otimes \beta}\right\rangle Y_{y \otimes \beta,(1,1) \otimes 1} \\
& =\sum_{x, y, \alpha, \beta}\left\langle i d, a_{x \otimes \alpha}\right\rangle Y_{x \otimes \alpha, y \otimes \beta} Y_{y \otimes \beta,(1,1) \otimes 1}
\end{aligned}
$$

where in the last step we have used Th. 5.7 of [BEK1] as in the proof of Prop. 3.1 in [BE4]. Since $Y_{\alpha \beta} Y_{\beta 1}=I_{H} \delta_{\alpha, 1}, Y_{x \otimes \alpha, y \otimes \beta}=Y_{x y} Y_{\alpha \beta}$, we get

$$
\begin{aligned}
\hat{c}=\sum_{x, y}\left\langle i d, a_{x \otimes 1}\right\rangle Y_{x y} Y_{y(1,1)} I_{H} & =\sum_{x, y}\langle(1,1), x\rangle Y_{x y} Y_{y 1} I_{H} \\
& =\sum_{y} Y_{(1,1) y} Y_{y 1} I_{H}=I_{G / H} I_{H} .
\end{aligned}
$$


It follows that $I_{\hat{W}}=I_{H} I_{G / H}$.

From the proof of the first equation above we have $v=c d, v^{-}=c d^{-}$, and hence $\left\langle v, v^{-}\right\rangle=I_{W_{0}} I_{W}^{2} / I_{W}^{2}$. But one can also compute directly that

$$
\left\langle v, v^{-}\right\rangle=\hat{c}=I_{G / H} I_{H}
$$

completing the proof of the third equality.

Now we are ready to prove the following:

Proposition 2.4. $\quad$ Suppose $G$ and $H$ are simply connected semisimple compact Lie groups of type $A$ as noted in Section 1. Assume $H \subset G_{k}$ is also cofinite. Then:

(1) $I_{G / H}=\mu_{G / H}$

(2) The $Y$ matrix of the coset is nondegenerate.

Proof. Ad (1): By the third equation in lemma $2.3 I_{W_{0}}=\frac{I_{H} I_{G / H}}{d(G / H)^{4}}$. On the other hand since $\sigma_{i} \in W_{0}, \forall i$ (cf. Lemma 3.5 of [X1] or [BE1]), it follows by definition that $I_{G}=\sum_{i} d_{\sigma_{i}}^{2} \leq I_{W_{0}}=\frac{I_{H} I_{G / H}}{d(G / H)^{4}}$. Since $I_{G}=\mu_{G}, I_{H}=\mu_{H}$, we get

$$
\mu_{G} \leq \frac{\mu_{H} I_{G / H}}{d(G / H)^{4}} .
$$

Note that $I_{G / H} \leq \mu_{G / H}$, but by Lemma 2.2

$$
\mu_{G}=\frac{\mu_{H} \mu_{G / H}}{d(G / H)^{4}} .
$$

It follows that all the $\leq$ above are $=$ and this proves $(1)$.

Ad (2): This follows immediately from (1), the second part of Th. 38 (note that strong additivity is not assumed) and Cor. 32 of [KLM].

A different proof of (2) of Lemma 2.4 without using the results of [KLM] can be given by using properties of relative braidings as follows. Let $x$ be an irreducible covariant representation of $\mathcal{A}_{G / H}$ which has trivial braidings with every covariant representation of $\mathcal{A}_{G / H}$, i.e., $\epsilon(x, y) \epsilon(y, x)=i d, \forall y$. Then $x \otimes 1$ is a covariant representation of $\mathcal{A}_{G / H} \otimes \mathcal{A}_{H}$ which has trivial braidings with every covariant representation of $\mathcal{A}_{G / H} \otimes \mathcal{A}_{H}$. It follows by definition that $a_{x \otimes 1}=\tilde{a}_{x \otimes 1}$, and so $a_{x \otimes 1} \in W_{0}$. Since $I_{W_{0}}=I_{G}$, as sectors $\left[a_{x \otimes 1}\right]=\left[\sigma_{i}\right]$ for some $i$ since $a_{x \otimes 1}$ is irreducible. Let $u$ be a unitary intertwinning operator such that $\sigma_{i}=u a_{x \otimes 1} u^{*}$. For any $\sigma_{j}$, since $\sigma_{j} \prec a_{(j, \beta) \otimes \beta}$, we can choose an isometry $u_{1}$ such that $\sigma_{j}=u_{1}^{*} a_{(j, \beta) \otimes \beta} u_{1}$. Note that by our assumption $x \otimes 1$ 
has trivial braidings with $(j, \beta) \otimes \beta$, and by using $u, u_{1}$ and the naturality of relative braidings (cf. Prop. 3.12, 3.15 of [BE3], also cf. Lemma 2.2.3 of [X6]), we conclude that $i$ and $j$ as irreducible covariant representations of $\mathcal{A}_{G}$ have trivial braidings. This forces $i$ to be identity, and it follows that $\left[a_{x \otimes 1}\right]=[i d]$ which implies that $x$ is the identity by Prop. 4.2 of [X4]. This proves (2) of Lemma 4.2 by the proposition in Section 5 of [Reh].

Corollary 2.5. $\quad$ Under the assumptions of Prop. $2.4 \mathcal{A}_{G / H}$ is n-regular for any $n \geq 1$.

Proof. This follows from Lemma 2.2, (1) of Lemma 2.4 and Cor. 7 of [KLM].

\section{$\S 3 . \quad$ Kac-Wakimoto Conjecture}

\section{$\S 3.1 . \quad$ Conjecture 2 of $[\mathrm{X} 4]$}

Let $H \subset G_{k}$ be as in Section 1. Throughout this section, we assume that $H \subset G_{k}$ is cofinite.

We will denote by $S, T$ (resp. $\dot{S}, \dot{T}$ ) the genus 0 modular matrices corresponding to $G$ (resp. $H$ ). As we remarked at the end of Section 2.1, they coincide with the genus 1 modular matrices when $G, H$ are type $A$.

We also assume that $H \subset G_{k}$ is not conformal, so the coset theory is nontrivial (cf. Prop. 2.2 of [X4]). But see the remark after Prop. 3.1 for the case of conformal inclusions.

By Prop. 2.4, the $Y$-matrix of the coset as defined in Section 2.1 is nondegenerate, and we shall denote by $\ddot{S}, \ddot{T}$ the corresponding genus 0 modular matrices. We will denote by $S, T$ (resp. $\dot{S}, \dot{T})$ the genus 0 modular matrices associated with $G$ (resp. $H$ ).

Throughout this section we will use genus 0 modular matrices only unless noted otherwise.

As in Section 2.2, we denote the set of irreducible sectors of $a_{x \otimes \alpha}$ by $W$. Notice $\sigma_{i} \in W$, and are referred to as "special nodes" in Section 3.4 of [X1]. Since (cf. [X4] or [BE1-2]) $a_{\bar{x} \otimes \bar{\alpha}}=\bar{a}_{x \otimes \alpha}, \sigma_{j} a_{x \otimes \alpha}=a_{x \otimes \alpha} \sigma_{j}$, the matrix corresponding to multiplications on $W$ by $\sigma_{i}, a_{x \otimes 1}$, and $a_{1 \otimes \alpha}$ are commuting normal matrices, so they can be simultaneously diagonalized. Note that all the irreducible representations of the ring generated by $\alpha^{\prime} s$ are given by (cf. Section 2.1 after (7))

$$
\alpha \rightarrow \frac{\dot{S}_{\alpha \beta}}{\dot{S}_{1 \beta}},
$$


and similarly for the ring generated by $\sigma_{i}^{\prime}$ s and $a_{x \otimes 1}$ 's, with $\dot{S}$ replaced by $S$ and $\ddot{S}$ respectively. Assume $\left\{\psi^{(k, \delta, z ; s)}\right\}$ are normalized orthogonal eigenvectors of the matrix corresponding to multiplications on $W$ by $\sigma_{i}, a_{x \otimes 1}$, and $a_{1 \otimes \alpha}$ with eigenvalues $\frac{S_{i k}}{S_{1 k}}, \frac{\ddot{S}_{x z}}{S_{1 z}}$ and $\frac{\dot{S}_{\alpha \delta}}{\dot{S}_{1 \delta}}$ respectively, $s$ is an index indicating the multiplicity of $k, \delta, z$, and we denote by $(\operatorname{Exp} 1)$ the set of $k, \delta, z ; s$ 's which appears in the set $\left\{\psi^{(k, \delta, z ; s)}\right\}$. Recall if a representation is denoted by 1 , it will always be the vacuum representation.

Lemma A. We have:

(1) The eigenvector $\psi^{(1,1,1 ; s)}$ is unique with multiplicity $s=1$, and is given by $\sum_{a} d_{a} a$, up to a positive constant; moreover $\sum_{a} d_{a}^{2}=\frac{1}{\left|\psi_{1}^{(1,1,1 ; 1)}\right|^{2}}$;

$$
\sum_{(k, \delta, z ; s) \in(E x p 1)} \frac{S_{\bar{i} k}}{S_{1 k}} \frac{\dot{S}_{\alpha \delta}}{S_{1 \delta}} \frac{\ddot{S}_{x z}}{\ddot{S}_{1 z}}\left|\psi_{1}^{(k, \delta, z ; s)}\right|^{2}=\left\langle\sigma_{i}, a_{x \otimes \alpha}\right\rangle
$$

(3) If

$$
\left\langle\sigma_{j}, a_{y \otimes \beta}\right\rangle \neq 0
$$

then $\omega_{y}=\omega_{j} \omega_{\beta}^{-1}$

(4)

$$
\sum_{j, \beta, y} S_{1 j} \overline{S_{1 \beta} \ddot{S_{1 y}}}\left\langle\sigma_{j}, a_{y \otimes \beta}\right\rangle=1 .
$$

Proof. Ad (1): Let $\tilde{G}=\sum_{x, \alpha} a_{x \otimes \alpha}$, then every element of $W$ appears as an irreducible subsector of $\tilde{G}$, and so we have

$$
\tilde{G}_{a b}:=\langle\tilde{G} a, b\rangle=\langle\tilde{G}, b \bar{a}\rangle>0
$$

since $W$ is a ring. It follows that $\left(\tilde{G}_{a b}\right)$ is an irreducible matrix and has up to positive constant a unique Perron-Frobenius eigenvector. But the vector $\sum_{a} d_{a} a$ is an eigenvector of $\left(\tilde{G}_{a b}\right)$ by the properties of statistical dimensions with maximal eigenvalue, and so up to positive constant the Perron-Frobenius eigenvector of $\left(\tilde{G}_{a b}\right)$ is $\sum_{a} d_{a} a$. Note that $\psi^{(1,1,1 ; s)}$ is an eigenvector of $\left(\tilde{G}_{a b}\right)$ with maximal eigenvalue, we must have $s=1$ and there exists a positive number $p$ such that $\psi_{a}^{(1,1,1 ; 1)}=p d_{a}, \forall a$. The last part of (1) now follows from $d_{1}=1$ and $\psi^{(1,1,1 ; 1)}$ is a unit vector. Note that there is an analogue statement in (3) of Th. 3.9 of $[\mathrm{X} 1]$.

$\operatorname{Ad}(2)$ : Note $\left\langle\sigma_{i}, a_{x \otimes \alpha}\right\rangle=\left\langle\sigma_{\bar{i}} a_{x \otimes \alpha}, 1\right\rangle$, and (2) follows from the definitions. Ad (3): By (4) of Th. 4.1 of [X4] (also cf. (2) of Prop. 4.2 of [X4]) we have

$$
\left\langle\sigma_{j}, a_{y \otimes \beta}\right\rangle=\langle(j, \beta), y\rangle,
$$


so if

$$
\left\langle\sigma_{j}, a_{y \otimes \beta}\right\rangle \neq 0,
$$

then $y$ appears as an irreducible sector of $(j, \beta)$. Note that the action of universal covering group $\mathbb{G}$ of $P S L(2, \mathbb{R})$ (cf. Prop. 2.2 of $[\mathrm{GL}]$ ) on the Hilbert space $H_{(j, \beta)}$ induces an action on the representation space $H_{y}$ corresponding to sector $y$, but the action of $2 \pi$ in $\mathbb{G}$ on the Hilbert space $H_{(j, \beta)}$ is given by a constant $\omega_{j} \omega_{\beta}^{-1}$, and it follows that the univalence $\omega_{y}$ of $y$ is given by $\omega_{y}=\omega_{j} \omega_{\beta}^{-1}$.

Ad (4): By local equivalence (cf. Th. B in Section 17 of [W2]), the minimal index of the subfactor $\pi^{j}\left(L_{I} H\right)^{\prime \prime} \vee\left(\pi^{j}\left(L_{I} H\right)^{\prime} \cap \pi^{j}\left(L_{I} G\right)^{\prime \prime}\right) \subset \pi^{j}\left(L_{I} G\right)^{\prime \prime}$ is independent of $j$, where $\pi^{j}$ is the representation corresponding to $j$. It follows from the properties of statistical dimensions (cf. [L6]) that

$$
\sum_{\beta} d_{(j, \beta)} d_{\beta}=d_{j}^{2} \sum_{\beta} d_{(1, \beta)} d_{\beta}=d_{j}^{2} d(G / H)^{2},
$$

where $d(G / H)^{2}=\sum_{\beta} d_{(1, \beta)} d_{\beta}$ and $d_{(j, \beta)}$ is the statistical dimension of the coset sector $(j, \beta)$. So we have:

$$
\begin{aligned}
\sum_{j, \beta, y} S_{1 j} \overline{S_{1 \beta} \ddot{S_{1 y}}}\left\langle\sigma_{j}, a_{y \otimes \beta}\right\rangle & =S_{11} \dot{S}_{11} \ddot{S}_{11} \sum_{j, \beta, y} d_{j} d_{\beta} d_{y}\langle(j, \beta), y\rangle \\
& =S_{11} \dot{S}_{11} \ddot{S}_{11} \sum_{j, \beta} d_{j} d_{(j, \beta)}=S_{11} \dot{S}_{11} \ddot{S}_{11} \sum_{j} d_{j}^{2} d(G / H)^{2} \\
& =\frac{d(G / H)^{2} \dot{S}_{11} \ddot{S}_{11}}{S_{11}} .
\end{aligned}
$$

Note that by our assumption $\mu_{G}=\frac{1}{S_{11}^{2}}, \mu_{H}=\frac{1}{S_{11}^{2}}$, and $\mu_{G / H}=I_{G / H}=\frac{1}{S_{11}^{2}}$ by (1) of Prop. 2.4, so it follows from Lemma 2.2 that

$$
\frac{d(G / H)^{2} \dot{S}_{11} \ddot{S}_{11}}{S_{11}}=1
$$

and the proof is complete.

Proposition 3.1.

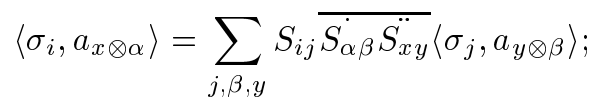

$$
\sum_{x}\left\langle\sigma_{i}, a_{x \otimes \alpha}\right\rangle \ddot{S}_{x z}=\sum_{j, \beta} S_{i j} \overline{S_{\alpha \beta}}\left\langle\sigma_{j}, a_{z \otimes \beta}\right\rangle .
$$


Proof. (2) Obviously follows from (1) and the unitarity of $\ddot{S}$, so we just need to prove (1). Use (cf. (2) and (3) of Section 2.1) we have

$$
\ddot{S_{x y}}=\sum_{w}\langle x y, w\rangle \frac{\omega_{x} \omega_{y}}{\omega_{w}} \tilde{S_{1 w}}
$$

so the right hand side of Prop. 3.1 is:

$$
\sum_{j, \beta, y, w} S_{i j} \overline{S_{\alpha \beta}}\left\langle\sigma_{j}, a_{y \otimes \beta}\right\rangle\langle x y, w\rangle \frac{\omega_{x}^{-1} \omega_{y}^{-1}}{\omega_{w}^{-1}} S_{1 w}^{.}
$$

Note by (3) of Lemma A that if

$$
\left\langle\sigma_{j}, a_{y \otimes \beta}\right\rangle \neq 0
$$

then $\omega_{y}=\omega_{j} \omega_{\beta}^{-1}$, so we can substitute $\omega_{j} \omega_{\beta}^{-1}$ for $\omega_{y}$ in the above expression. Now take the complex conjugate of both sides of (2) of Lemma A with $(i, \alpha, x)$ replaced by $(j, \beta, y)$ we have:

$$
\left\langle\sigma_{j}, a_{y \otimes \beta}\right\rangle=\sum_{k, \delta, z ; s} \frac{S_{j k}}{S_{1 k}} \frac{\dot{S}_{\bar{\beta} \delta}}{\dot{S}_{1 \delta}} \frac{\ddot{S}_{\bar{y} z}}{\ddot{S}_{1 z}}\left|\psi_{1}^{(k, \delta, z ; s)}\right|^{2},
$$

and we shall plug this into $(*)$ and we shall call this resulting expression after the two substitutions above by $\left(^{*}\right)$ in the following. We first sum over $j$ and $\beta$ in $\left(^{*}\right)$ using (cf. (5) of Section 2.1)

$$
\begin{aligned}
& \sum_{j} \omega_{j}^{-1} S_{i j} S_{j k}=C^{3} \omega_{i} \omega_{k} S_{\bar{i} k}, \\
& \sum_{\beta} \omega_{\beta} \dot{S}_{\alpha \beta} \dot{S}_{\beta \delta}=\dot{C}^{-3} \omega_{\alpha}^{-1} \omega_{\delta}^{-1} \dot{S}_{\alpha \delta},
\end{aligned}
$$

and then sum over $y$ in $(*)$ using (cf. (2) of Section 2.1)

$$
\sum_{y}\langle x y, w\rangle \frac{\ddot{S}_{\bar{y} z}}{\ddot{S}_{1 z}}=\frac{\ddot{S}_{x z}}{\ddot{S}_{1 z}} \frac{\ddot{S}_{\bar{w} z}}{\ddot{S}_{1 z}},
$$

and finally sum over $w$ in (*) using (cf. (5) of Section 2.1)

$$
\sum_{w} \ddot{S}_{1 w} \omega_{w} \ddot{S}_{w z}=\ddot{C}^{-3} \omega_{z}^{-1} \ddot{S}_{1 z}
$$

The right hand side of Prop. 3.1 is then

$$
\sum_{(k, \delta, z ; s) \in(E x p 1)} \frac{C^{3}}{\dot{C}^{3} \ddot{C}^{3}} \frac{\dot{\omega}_{i}}{\omega_{\alpha} \omega_{x}} \frac{\dot{\omega}_{k}}{\omega_{\delta} \omega_{z}} \frac{S_{\bar{i} k}}{S_{1 k}} \frac{\dot{S}_{\alpha \delta}}{S_{1 \delta}} \frac{\ddot{S}_{x z}}{\ddot{S}_{1 z}}\left|\psi_{1}^{(k, \delta, z ; s)}\right|^{2} .
$$


Set

$$
i=1, \alpha=1, x=1,
$$

and use (4) of Lemma A, we have:

$$
\sum_{(k, \delta, z ; s) \in(E x p 1)} \frac{C^{3}}{\dot{C}^{3} \ddot{C}^{3}} \frac{\omega_{k}}{\omega_{\delta} \omega_{z}}\left|\psi_{1}^{(k, \delta, z ; s)}\right|^{2}=1 .
$$

By setting $i=1, \alpha=1, x=1$ in (2) of Lemma A we have

$$
\sum_{(k, \delta, z ; s) \in(E x p 1)}\left|\psi_{1}^{(k, \delta, z ; s)}\right|^{2}=1 .
$$

Since

$$
\left|\frac{C^{3}}{\dot{C}^{3} \ddot{C}^{3}}\right|=\left|\frac{\omega_{k}}{\omega_{\delta} \omega_{z}}\right|=1,
$$

and $|a+b| \leq|a|+|b|, \forall a, b \in \mathbb{C}$, we must have

$$
\left.|| \psi_{1}^{(1,1,1 ; 1)}\right|^{2}+\left.\frac{\omega_{k}}{\omega_{\delta} \omega_{z}}\left|\psi_{1}^{(k, \delta, z ; s)}\right|^{2}|=| \psi_{1}^{(1,1,1 ; 1)}\right|^{2}+\left|\psi_{1}^{(k, \delta, z ; s)}\right|^{2}, \forall(k, \delta, z ; s),
$$

and since by (1) of Lemma A

$$
\left|\psi_{1}^{(1,1,1 ; 1)}\right|^{2}>0
$$

we have

$$
\frac{\omega_{k}}{\omega_{\delta} \omega_{z}}\left|\psi_{1}^{(k, \delta, z ; s)}\right|^{2}=\left|\psi_{1}^{(k, \delta, z ; s)}\right|^{2},
$$

and it follows that if

$$
\left|\psi_{1}^{(k, \delta, z ; s)}\right| \neq 0
$$

for some $s$, then $\frac{\omega_{k}}{\omega_{\delta} \omega_{z}}=1$.

It follows that $\frac{C^{3}}{\dot{C}^{3} \ddot{C}^{3}}=1$ and we have proved that the RHS of Prop. 3.1 is:

$$
\sum_{k, \delta, z ; s} \frac{S_{\bar{i} k}}{S_{1 k}} \frac{\dot{S}_{\alpha \delta}}{S_{1 \delta}} \frac{\ddot{S}_{x z}}{\ddot{S}_{1 z}}\left|\psi_{1}^{(k, \delta, z ; s)}\right|^{2}
$$

which is precisely the left hand side by (2) of Lemma A.

Note that $\frac{C^{3}}{\dot{C}^{3} \ddot{C}^{3}}=1$ shows that $\ddot{C}^{3}=\frac{C^{3}}{\dot{C}^{3}}=\exp \left(-6 \pi i \frac{\left(C_{G}-C_{H}\right)}{24}\right)$, where $C_{G}, C_{H}$ are central charges as defined in (11) of Section 2.1. This matches with the result of [GKO] that the central charge of the coset is $C_{G}-C_{H}$. For the special of diagonal cosets of type A, this is (2) of Th. 2.3 in [X5] which is proved by different methods. 
Note that if $H \subset G_{1}$ is a conformal inclusion, then by a similar but simpler proof as above one can show the following:

$$
b_{i \alpha}=\sum_{j, \beta} S_{i j}{\dot{S_{\alpha \beta}}}_{j \beta},
$$

where $b_{i \alpha} \in \mathbb{N}$ are the branching coefficients. This is implied by (1) of Th. A on P. 185 of $[\mathrm{KW}]$.

By setting $i=1, \alpha=1, x=1$ in (1) of Prop. 3.1 we have:

$$
\begin{aligned}
1 & =\sum_{j, \beta, y} S_{1 j} \overline{\dot{S}_{1 \beta} \ddot{S}_{1 y}}\left\langle\sigma_{j}, a_{y \otimes \bar{\beta}}\right\rangle \\
& =\sum_{s} \frac{1}{S_{11} \dot{S}_{11} \ddot{S}_{11}}\left|\psi^{(1,1,1 ; s)}\right|^{2},
\end{aligned}
$$

so it follows from (1) of Lemma A that

$$
\sum_{a \in W} d_{a}^{2}=\frac{1}{S_{11} \dot{S}_{11} \ddot{S}_{11}},
$$

where $a \in W$ means the summation over the basis of $W$ given by irreducible sectors.

In [X5] we define $V$ to be the vector space whose basis are irreducible subsectors of $\sigma_{i} a_{1 \otimes \alpha}$, and $\psi^{(j, \beta, s)}$ are normalized eigenvectors of linear transformations on $V$ which are multiplications by $\sigma_{i}$ and $a_{1 \otimes \alpha}$. The proof of (1) of Lemma A applies in this case with $\tilde{G}=\sum_{i, \alpha} \sigma_{i} a_{1 \otimes \alpha}$ and we have

$$
\sum_{a \in V} d_{a}^{2}=\frac{1}{S_{11} \dot{S}_{11} b^{0}(1,1)}
$$

where (cf. Prop. 3.1 of [X5])

$$
b^{0}(1,1):=\sum_{j, \beta} S_{1 j} \dot{S}_{1 \beta}\left\langle\sigma_{j}, a_{1 \otimes \beta}\right\rangle .
$$

Set $i=1, \alpha=1, z=1$ in (2) of Prop. 3.1 we have

$$
b^{0}(1,1)=\ddot{S}_{11} .
$$

So we have proved that

$$
\sum_{a \in W} d_{a}^{2}=\sum_{a \in V} d_{a}^{2}
$$


and since $V \subset W$, we must have $V=W$. So for any irreducible sector $x$ of the coset, there exists $(j, \beta)$ such that $a_{x \otimes 1}$ is an irreducible subsector of $\sigma_{j} a_{1 \otimes \bar{\beta}}$. Since

$$
\left\langle\sigma_{j} a_{1 \otimes \bar{\beta}}, a_{x \otimes 1}\right\rangle=\left\langle\sigma_{j}, a_{x \otimes \beta}\right\rangle=\langle(j, \beta), x\rangle,
$$

we have proved the following:

Corollary 3.2. $\quad$ Every irreducible sector of the coset appears as an irreducible subsector of some $(j, \beta) \in \exp$.

Note (1) of Th. 2.3 in [X5] follows from Cor. 3.2 above and Cor. 32 of $[\mathrm{KLM}]$.

Cor. 3.2 proves a stronger version of Conj. 1 in [X4] under the conditions stated at the beginning of this section. It is interesting to note that there is also a Vertex Operator Algebra (VOA) approach to the coset CFT in [FZ]. In Section 5 of [FZ] (also cf. P. 113 of [Kacv]) a coset VOA is defined and it is conjectured that these coset VOA is rational (cf. [Z] for definitions). For $(j, \beta) \in \exp$, and $x$ a subsector of $(j, \beta)$, let $H_{(j, \beta)}, H_{x} \subset H_{(j, \beta)}$ be the corresponding Hilbert spaces of representations. It is easy to see using Section 2.3 of [X4] that $H_{x}$ is also an irreducible representation of the coset VOA. This shows Th. 4.3 of [X4] and Lemma 2.2 of [X5] holds for the coset VOA in the case of diagonal coset of type A, which is a result that has not been proved by using the theory of VOA so far. However to use Cor. 3.2 to prove the rationality of the coset VOA, one has to show that any representation of this coset VOA admits a natural inner product so similar norm estimations as in Section 2.3 of [X4] can be carried out. ${ }^{7}$

More generally let us define

$$
b^{0}(i, \alpha):=\sum_{j, \beta} S_{i j} \bar{S}_{\alpha \beta}\left\langle\sigma_{j}, a_{1 \otimes \beta}\right\rangle .
$$

Note as stated at the beginning of this section that all the $S, \dot{S}$ matrices above are genus $0 S, \dot{S}$ matrices. So $b^{0}(i, \alpha)$ is defined differently from $b(i, \alpha)$ in (1) of Section 1 where genus $1 S, \dot{S}$ matrices are used.

Corollary 3.3. The statistical dimension $d_{(i, \alpha)}$ of the sector $(i, \alpha)$ is given by

$$
d_{(i, \alpha)}=\frac{b^{0}(i, \alpha)}{b^{0}(1,1)} .
$$

Proof. By setting $i=1, \alpha=1, z=1$ in (2) of Prop. 3.1 we have $b^{0}(1,1)=$ 
$\ddot{S}_{11}$, and by setting $z=1$ in (2) of Prop. 3.1 we have

$$
\begin{aligned}
\frac{b^{0}(i, \alpha)}{b^{0}(1,1)} & =\sum_{x}\left\langle\sigma_{i}, a_{x \otimes \alpha}\right\rangle \frac{\ddot{S}_{x 1}}{\ddot{S}_{11}} \\
& =\sum_{x}\left\langle\sigma_{i}, a_{x \otimes \alpha}\right\rangle d_{x} \\
& =\sum_{x}\langle(i, \alpha), x\rangle d_{x} \\
& =d_{(i, \alpha)}
\end{aligned}
$$

which completes the proof of the Corollary.

Note that if the genus $1 S$ (resp. $\dot{S}$ ) matrix corresponding to $G$ (resp. $H$ ) coincide with the genus $0 S$ (resp. $\dot{S}$ ) corresponding to $G$ (resp. $H$ ), then $b^{0}(i, \alpha)$ coincides with $b(i, \alpha)$ defined in (1) of Section 1. By Cor. 3.3, we have proved the following theorem:

Theorem 3.4. $\quad$ Suppose $G$ and $H$ are simply connected semisimple compact Lie groups of type $A$ as noted in Section 1. Assume $H \subset G_{k}$ is also cofinite.

Then Conjecture 2 in $[\mathrm{X} 4]$ is true, i.e., the statistical dimension $d_{(i, \alpha)}$ of the coset sector $(i, \alpha)$ is given by

$$
d_{(i, \alpha)}=\frac{b(i, \alpha)}{b(1,1)} .
$$

Since $d_{(i, \alpha)} \geq 1, b(1,1)>0$, an immediate corollary of Th. 3.4 is the following:

Corollary 3.5. Under the same conditions of Theorem 3.4, the KacWakimoto Conjecture is true, i.e., if $(i, \alpha) \in \exp$, then $b(i, \alpha)>0$.

Let us mention some examples which satisfy the assumptions of Th. 3.4. Take $H_{l} \subset G_{1}$ to be conformal inclusion where $H, G$ are simply connected type $A$ Lie groups. Here is a list of such pairs:

$$
\begin{aligned}
& S U(N)_{N-2} \subset S U\left(\frac{N(N-1)}{2}\right), N \geq 4 ; \\
& S U(N)_{N+2} \subset S U\left(\frac{N(N+1)}{2}\right), \\
& S U(M)_{N} \times S U(N)_{M} \subset S U(N M) .
\end{aligned}
$$

Consider the coset $H_{l k} \subset G_{k}$ with $k \geq 2$. By [W2] and [X3], $H_{l k}, G_{k}$ are $\mu$ rational and the genus $0 \mathrm{~S}$-matrices coincide with the genus $1 \mathrm{~S}$-matrix, and 
by (2) of Cor. 3.1 of [X4] the coset $H_{l k} \subset G_{k}$ is cofinite, so the conclusion of Th. 3.4 and Cor. 3.5 is true in these examples. To the best of our knowledge, this is already a new result since the branching rules (the set exp) is not known in general ${ }^{8}$ for these examples, and even with the explict formula for exp, the calculation of $b(i, \alpha)$ seems to be nontrivial in general.

\section{§3.2. Conjecture 7.1 of [BE3]}

We shall use the original settings of [X1]. Let $H_{k} \subset G_{1}$ be a conformal inclusion with both $G$ and $H$ being semisimple compact Lie groups of type $A$, and $k$ the Dynkin index of the inclusion (cf. [KW]). We use $i$ (resp. $\lambda$ ) to denote the irreducible projective positive energy representation of loop group $L G$ (resp. $L H)$ at level 1 (resp. k) (cf.[PS]).

Denote by $b_{i \lambda}$ the branching coefficients, i.e., when restricting to $L G, i$ decomposes as $\sum_{\lambda} b_{i \lambda} \lambda$. Denote by $S_{i j}$ (resp. $S_{\lambda \mu}$ ) the genus 1 S-matrices of $L G$ (resp. $L H$ ) at level 1 (resp. k) (cf. [Kac]). Recall $a_{\lambda}$ as defined on Page 372 of $[\mathrm{X} 1]$, then we have (cf. P. 9 of [X2])

$$
b_{i \lambda}=\left\langle a_{\lambda}, \sigma_{i}\right\rangle .
$$

Let us first prove a lemma in the setting of [X1] which is an analogue of Lemma 3.10 of [BE3]. The basic idea is already implicit in the proof of Lemma 3.2 in [X1]. The proof depends on Section 3 of [X1] and we refer the reader to [X1] for unexplained notations.

Lemma 3.6. If $\left\langle a_{\lambda}, \tilde{a}_{\mu}\right\rangle \neq 0$, then $\omega_{\lambda}=\omega_{\mu}$.

Proof. Let $u \neq 0$ be in $\operatorname{Hom}\left(a_{\lambda}, \tilde{a}_{\mu}\right)$. Then

$$
\rho(u) \in \operatorname{Hom}(\gamma \lambda, \gamma \mu)=\operatorname{Hom}\left(\rho a_{\lambda} \bar{\rho}, \rho a_{\mu} \bar{\rho}\right),
$$

so $u \in \operatorname{Hom}\left(a_{\lambda} \bar{\rho}, a_{\mu} \bar{\rho}\right)$, and by (1) of Th. 3.3 in [X1], we have $u \in \operatorname{Hom}\left(a_{\lambda}, a_{\mu}\right)$. So we get:

$$
a_{\mu}(m) u=\tilde{a}_{\mu}(m) u, \forall m \in M .
$$

Set $m=w$, apply $\rho$ to both sides, and use the equation on P. 373 of [X1] we obtain

$$
\gamma(\sigma) w \rho(u)=\gamma(\tilde{\sigma}) w \rho(u) .
$$

Multiply on the left by $v^{*}$ and use the equation on P. 369 of [X1] we get:

$$
\begin{aligned}
& v^{*} \gamma(\sigma) w \rho(u)=d_{\rho}^{-1} \sigma \rho(u) \\
& v^{*} \gamma(\tilde{\sigma}) w \rho(u)=d_{\rho}^{-1} \tilde{\sigma} \rho(u)
\end{aligned}
$$


and so

$$
\sigma \rho(u)=\tilde{\sigma} \rho(u) .
$$

Note this equation is an analogue of Lemma 3.6 of [BE3].

Now multiply both sides on the right by $v$, we have

$$
\sigma \rho(u) v=\tilde{\sigma} \rho(u) v
$$

hence

$$
\sigma^{-1} \tilde{\sigma} \rho(u) v=\rho(u) v .
$$

Note that $\rho(u) v \in \operatorname{Hom}(\lambda, \gamma \mu)$, and apply the monodromy equation (cf. P. 359 of [X1] and use the fact that the univalence of $\gamma$ is 1) we get:

$$
\omega_{\lambda} \omega_{\mu}^{-1} \rho(u) v=\rho(u) v .
$$

To finish the proof we just have to show that $\rho(u) v$ is not zero. Note by (3) of Prop. 2.6 of [X1] we have $\rho\left(u^{*}\right)=\gamma\left(u_{1}\right) w$ for some $u_{1} \in M$, and so $\rho(u) v=w^{*} \gamma\left(u_{1}^{*}\right) v=w^{*} v \gamma\left(u_{1}^{*}\right)=d_{\rho}^{-1} \gamma\left(u_{1}^{*}\right)$, so if $\rho(u) v=0$, then $\rho(u)=0$, and so $u=0$ contradicting our assumption $u \neq 0$.

Let $U$ be the vector space with a basis which consists of irreducible components of $a_{\lambda} \tilde{a}_{\mu} \sigma_{i}, \forall \lambda, \mu, i . a_{\lambda}, \tilde{a}_{\mu}, \sigma_{i}$ acts on $U$ by multiplication, and since they are normal commuting matrices by (2) of Cor. 3.5 and Lemma 3.3 of [X1], they can be simultaneously diagonalized, and suppose $\left\{\psi^{\left(j, \lambda_{1}, \mu_{1} ; s\right)}\right\}$ are normalized orthogonal eigenvectors of $a_{\lambda}, \tilde{a}_{\mu}$, and $\sigma_{i}$ with eigenvalues $\frac{\dot{S}_{\lambda \lambda_{1}}}{\dot{S}_{1 \lambda_{1}}}, \frac{\dot{S}_{\mu \mu_{1}}}{\dot{S}_{1 \mu_{1}}}$, and $\frac{S_{i j}}{S_{1 j}}$ respectively, where $s$ is an index indicating the multiplicity of $j, \lambda_{1}, \mu_{1}$.

Theorem 3.7.

$$
\left\langle\tilde{a}_{\mu}, a_{\lambda}\right\rangle=\sum_{i} b_{i \mu} b_{i \lambda}
$$

Proof. Let us calculate

$$
\sum_{\lambda, \mu} \dot{S}_{1 \mu}\left\langle\tilde{a}_{\mu}, a_{\lambda}\right\rangle \dot{S}_{1 \lambda}
$$

as in the proof of Prop. 3.1. By Lemma 3.6, we have

$$
\begin{aligned}
\sum_{\lambda, \mu} \dot{S}_{1 \mu}\left\langle\tilde{a}_{\mu}, a_{\lambda}\right\rangle \dot{S}_{1 \lambda} & =\sum_{\lambda, \mu} \dot{S}_{1 \mu} \frac{\omega_{\lambda}}{\omega_{\mu}}\left\langle\tilde{a}_{\mu}, a_{\lambda}\right\rangle \dot{S}_{1 \lambda} \\
& =\sum_{\lambda, \mu, i, \lambda_{1}, \mu_{1} ; s} \dot{S}_{1 \mu} \omega_{\mu}^{-1} \dot{S}_{\bar{\mu} \mu_{1}} \dot{S}_{1 \lambda} \omega_{\lambda} \dot{S}_{\lambda \lambda_{1}} \frac{1}{\dot{S}_{1 \lambda_{1}} \dot{S}_{1 \mu_{1}}}\left|\psi_{1}^{\left(i, \lambda_{1}, \mu_{1} ; s\right)}\right|^{2} \\
& =\sum_{i, \lambda_{1}, \mu_{1} ; s} \omega_{\lambda_{1}}^{-1} \omega_{\mu_{1}}\left|\psi_{1}^{\left(i, \lambda_{1}, \mu_{1} ; s\right)}\right|^{2}
\end{aligned}
$$


where we have used (5) of Section 2.1 in the last $=$. It follows that

$$
\sum_{\lambda, \mu} \dot{S}_{1 \mu}\left\langle\tilde{a}_{\mu}, a_{\lambda}\right\rangle \dot{S}_{1 \lambda} \leq \sum_{i, \lambda_{1}, \mu_{1} ; s}\left|\psi_{1}^{\left(i, \lambda_{1}, \mu_{1} ; s\right)}\right|^{2}=\langle 1,1\rangle=1 .
$$

On the other hand

$$
\begin{aligned}
\sum_{\lambda, \mu} \dot{S}_{1 \mu}\left\langle\tilde{a}_{\mu}, a_{\lambda}\right\rangle \dot{S}_{1 \lambda} & \geq \sum_{\lambda, \mu, i} \dot{S}_{1 \mu}\left\langle\tilde{a}_{\mu}, \sigma_{i}\right\rangle\left\langle\sigma_{i}, a_{\lambda}\right\rangle \dot{S}_{1 \lambda} \\
& =\sum_{\lambda, \mu, i} \dot{S}_{1 \mu} b_{i \mu} b_{i \lambda} \dot{S}_{1 \lambda} \\
& =\sum_{i} S_{i 1} S_{1 i}=1
\end{aligned}
$$

where in the second $=$ we have used $(\mathrm{a})$ of Th. A on P. 185 of [KW]. So we must have

$$
\sum_{\lambda, \mu} \dot{S}_{1 \mu}\left\langle\tilde{a}_{\mu}, a_{\lambda}\right\rangle \dot{S}_{1 \lambda}=\sum_{\lambda, \mu, i} \dot{S}_{1 \mu} b_{i \mu} b_{i \lambda} \dot{S}_{1 \lambda}
$$

and since

$$
\left\langle\tilde{a}_{\mu}, a_{\lambda}\right\rangle \geq \sum_{i} b_{i \mu} b_{i \lambda}
$$

and $\dot{S}_{1 \mu}>0, \dot{S}_{1 \lambda}>0$, we must have

$$
\left\langle\tilde{a}_{\mu}, a_{\lambda}\right\rangle=\sum_{i} b_{i \mu} b_{i \lambda}
$$

Th. 3.6 proves Conj. 7.1 of [BE3] is true. This together with Prop. 5.1 of [BE3] show that the invariants of the dual Jones-Wassermann subfactors associated with conformal inclusions are determined by the ring structure generated by irreducible sectors of $a_{\lambda} \tilde{a}_{\mu}$, thus removing the mystery expressed in the footnote on P. 393 of [X1], where one can also find the first example of such ring.

\section{Footnotes}

1 When $\mathrm{G}$ is the direct product of simple groups, $k$ is a multi-index, i.e., $k=\left(k_{1}, \ldots, k_{n}\right)$, where $k_{i} \in \mathbb{N}$ corresponding to the level of the $i$-th simple group. The level of $L H$ is determined by the Dynkin indices of $H \subset G$. To save some writing we write the coset as $H \subset G_{k}$.

2 This is slightly different from the notation $\pi^{0}$ (resp. $\pi_{0}$ ) in [X4]: it seems to be more appropriate since these representations correspond to identity sectors.

$3 \operatorname{Our}(j, \beta)$ corresponds to $(M, \mu)$ on P.186 of [KW], and it follows from the definition (cf. Section 2.1) and Cor. 2.10 of [GL] that $\langle(j, \beta),(1,1)\rangle$ is then equal to mult $_{M}(\mu, p)$ which appears in 2.5.4 of [KW] if the sector $(j, \beta)$ has finite index, and that our formula (1) is identical to 2.5 .4 of $[\mathrm{KW}]$. 
4 After this paper appears as preprint math.RT/9904098 in the net, the author is informed by Prof. D. Evans that Conj. 7.1 is also proved in a forthcoming paper (cf. [BEK1]) by different methods.

5 As in [GL] by an interval of the circle we mean an open connected proper subset of the circle. If $I$ is such an interval then $I^{\prime}$ will denote the interior of the complement of $I$ in the circle.

6 Note this is slightly different from the definition of vector space $V$ in Section 3.1 of [X5], and in fact $V \subset W$, but we will see in Cor. 3.2 that these two spaces coincide.

7 We'd like to thank Dr. Yongchang Zhu for a discussion on this point.

8 The branching rules in the case of conformal inclusions listed here are the main results of $[\mathrm{LL}]$ and $[\mathrm{ABI}]$ and are by no means trivial.

\section{References}

[ABI] Altschuler, D., Bauer, M. and Itzykson, C., The branching rules of conformal embeddings, Comm. Math. Phys., 132 (1990), 349-364.

[BE1] Böckenhauer, J. and Evans, D. E., Modular invariants, graphs and $\alpha$-induction for nets of subfactors. I, Comm. Math. Phys., 197 (1998), 361-386.

[BE2] — Modular invariants, graphs and $\alpha$-induction for nets of subfactors. II, Comm. Math. Phys., 200 (1999), 57-103.

[BE3] - Modular invariants, graphs and $\alpha$-induction for nets of subfactors. III, Comm. Math. Phys., 205 (1999), 183-228. Also see hep-th/9812110.

[BE4] — Modular Invariants from Subfactors: Type I Coupling Matrices and Intermediate Subfactors, math. OA/9911239.

[BEK1] Böckenhauer, J., Evans, D. E. and Kawahigashi, Y., On $\alpha$-induction, chiral generators and modular invariants for subfactors, Comm. Math. Phys., 208 (1999), 429-487. Also see math. OA/9904109.

[BEK2] - Chiral structure of modular invariants for subfactors, Comm. Math. Phys., 210 (2000), 733-784.

[BAF] Buchholz, D., D'Antoni, C. and Fredenhagen, K., Local factorizations. The universal structure of local algebras, Comm. Math. Phys., 111 (1987), 123-135.

[FRS] Fredenhagen, K., Rehren, K.-H. and Schroer, B., Superselection sectors with braid group statistics and exchange algebras. II, Rev. Math. Phys., Special issue (1992), $113-157$.

[FZ] Frenkel, I. and Zhu, Y., Vertex operator algebras associated to representations of affine and Virasoro algebras, Duke Math. J., 66 (1992), 123-168.

[GL] Guido, D. and Longo, R., The Conformal Spin and Statistics Theorem, Comm. Math. Phys., 181 (1996), 11-35.

[GKO] Goddard, P. and Olive, D. eds., Kac-Moody and Virasoro algebras, Adv. Ser. Math. Phys., 3 (1988).

[J] Jones, V., Fusion en alǵebres de Von Neumann et groupes de lacets (d'aprés A. Wassermann), Astérisque, 237 (1996), 251-273.

[KLM] Kawahigashi, Y., Longo, R. and Müger, M., Multi-interval Subfactors and Modularity of Representations in Conformal Field theory, Preprint 1999, see also math. OA/9903104.

[KW] Kac, V. G. and Wakimoto, M., Modular and conformal invariance constraints in representation theory of affine algebras, Adv. Math., 70 (1988), 156-234.

[Kac] Kac, V. G., Infinite Dimensional Lie Algebras, 3rd Edition, Cambridge University Press, 1990.

[Kacv] - Vertex algebras for beginners, AMS, 1998.

[Ka] Kawahigashi, Y., Classification of paragroup actions on subfactors, Publ. RIMS, Kyoto Univ., 31 (1995), 481-517. 
[L1] Longo, R., Von Neumann Algebras and Quantum Field Theory, Proceedings of International Congress of Mathematicians, Aug. 3-11, 1994, Zürich, (1995), 1281-1291.

[L2] — Duality for Hopf algebras and for subfactors, I, Comm. Math. Phys., 159 (1994), 133-150.

[L3] - Index of subfactors and statistics of quantum fields, I, Comm. Math. Phys., 126 (1989), 217-247.

[L4] - Index of subfactors and statistics of quantum fields, II, Comm. Math. Phys., 130 (1990), 285-309.

[L5] — An analogue of the Kac-Wakimoto formula and black hole conditional entropy, gr-qc 9605073, to appear in Comm. Math. Phys.

[L6] - Minimal index and braided subfactors, J. Funct. Anal., 109 (1992), 98-112.

[LL] Levstein, F. and Liberati, J. I., Branching Rules for Conformal Embeddings, Comm. Math. Phys., 173 (1995), 1-16.

[LR] Longo, R. and Rehren, K.-H., Nets of subfactors, Rev. Math. Phys., 7 (1995), 567597.

[MS] Moore, G. and Seiberg, N., Taming the conformal zoo, Lett. Phys. B, 220 (1989), 422-430.

[PP] Pimsner, M. and Popa, S., Entropy and index for subfactors, Ann. Sci. Éc. Norm. Sup., 19 (1986), 57-106.

[PS] Pressley, A. and Segal, G., Loop Groups, Oxford University Press, 1986.

[Reh] Karl-Henning Rehren, Braid group statistics and their superselection rules, in, The algebraic theory of superselection sectors, World Scientific, 1990.

[Reh2] — Chiral observables and modular invariants, hep-th/9903262.

[TL] Toledano Laredo, V., Fusion of Positive Energy Representations of $\operatorname{LSpin}_{2 n}$, Ph. D. dissertation, University of Cambridge, 1997

[W1] Wassermann, A., Operator Algebras and Conformal Field Theory, Proceedings of International Congress of Mathematicians, Aug. 3-11, 1994, Züirich, (1995), 966979.

[W2] — Operator algebras and Conformal field theories III, Invent. Math., 133 (1998), 467-539.

[W3] - Operator algebras and Conformal field theories, preliminary notes of 1992.

[W4] Wassermann, A., with contributions by Jones, V., Lectures on operator algebras and conformal field theory, Proceedings of Borel Seminar, Bern 1994, to appear.

[We] Wenzl, H., Hecke algebras of type A and subfactors, Invent. Math., 92 (1988), 345383.

[X1] Xu, F., New braided endomorphisms from conformal inclusions, Comm. Math. Phys., 192 (1998), 349-403.

[X2] — Applications of braided endomorphisms from conformal inclusions, Inter. Math. Res. Notice., 1 (1998), 5-23, also see q-alg/9708013, and Erratum, Inter. Math. Res. Notice., 8 (1998).

[X3] — Jones-Wassermann subfactors for Disconnected Intervals, Comm. Contemp. Math., 2 (2000), 307-347.

[X4] — Algebraic coset conformal field theories, Comm. Math. Phys., 211 (2000), $1-43$.

[X5] - Algebraic coset conformal field theories II, Publ. RIMS, Kyoto Univ., 35 (1999), 795-824.

[X6] - 3-manifold invariants from cosets, math. GT/9907077.

[Z] Zhu, Y., Modular invariance of characters of vertex operator algebras, $J$. Amer. Math. Soc., 9 (1996), 237-302. 